\title{
表面固载的蛋白质对银纳米粒子的吸附及蛋白质 固载的可视化检测
}

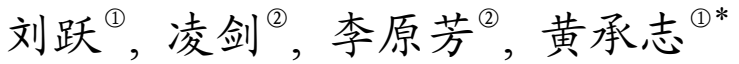 \\ (1) 西南大学药学院, 发光与实时分析教育部重点实验室, 重庆 400715; \\ (2) 西南大学化学化工学院, 发光与实时分析教育部重点实验室, 重庆 400715 \\ * 通讯作者, E-mail: Chengzhi@swu.edu.cn
}

收稿日期：2008-08-26；接受日期：2008-12-03

\begin{abstract}
摘要银纳米粒子有强烈的等离子体共振散射特性, 可作为光散射光谱探针用于 示踪和可视化分析. 通过研究固载于载玻片上的蛋白质对银纳米粒子的吸附, 发现 吸附在载玻片上的银纳米粒子在普通的白光发光二极管(LED)照射下, 肉眼就能看 见其散射光. 据此建立可视化检测蛋白质在载玻片上最小完全固载量的方法.
\end{abstract}

\section{关键词}

光散射

吸附机理

最小完全固载量

可视化
两性生物大分子蛋白质在不同酸度条件下带有 不同的电荷，与带相反电荷的基体能够发生吸附作 用. 而常规合成的金、银纳米粒子表面多包被有如柠 檬酸根等稳定剂, 所以在特定酸度的溶液中蛋白质 与金、银纳米粒子能够发生吸附. 基于两者的吸附作 用, 问海刚研究了血清白蛋白对银纳米粒子的包覆 作 ${ }^{[1]}$; Jing等人利用表面增强拉曼散射(SERS)和密度 功能理论(DFT)研究了 L-赖氨酸与金、银纳米粒子的 吸附过程 ${ }^{[2]}$. 但其吸附的有关参数及详细机理还需要 使用不同的手段加以探讨.

共振光散射分析法只需要在普通荧光分光光度 计上测定光散射信号, 因而其操作简便并且灵敏度 高 $^{[3]}$. 近年来已在蛋白质 ${ }^{[4]}$ 、核酸 ${ }^{[5]}$ 及核酸杂交 ${ }^{[6]}$ 、药 物 $^{[7]}$ 、染料 ${ }^{[8]}$ 等分析测试领域得到广泛应用. 金、银 纳米粒子是理想的光散射光谱探针,可作为荧光类似 物应用于细胞生物学、免疫及DNA分析等 ${ }^{[9]}$. 如金纳 米材料修饰抗体用于癌细胞的散射成像诊断 ${ }^{[10,11]}$ 和 肿瘤的光热治疗 ${ }^{[11]}$. 蒋治良等利用标记有抗体的银 纳米粒子通过共振光散射法检测纤维蛋白原 ${ }^{[12]}$.
在蛋白质检测方法上，常用的银染法具有很高 的灵敏度 ${ }^{[13]}$, 但同时其具有操作繁琐、污染会极大降 低其灵敏度等缺点 ${ }^{[14]}$. 本文拟利用银纳米粒子的等 离子体光散射特性对其与蛋白质的吸附机理进行探 讨, 并建立基于光散射信号的可视化分析检测蛋白 质固载方法. 该研究方法通过银纳米粒子散射光的 可见性对蛋白质在一定面积载玻片上的固载进行了 可视化检测研究, 实现了对蛋白质固载的快速、简便 测定.

\section{1 实验部分}

\section{1 试剂}

$1.0 \mathrm{mg} / \mathrm{mL}$ 人血清白蛋白(HAS, Sigma, $96.99 \%$ )、 牛血清白蛋白 $\mathrm{BSA}$, 上海长阳生化制药厂, 电泳单 点纯)、纤维素酶(Cellulase, 上海东风生化技术公司, 生化纯)、糜蛋白酶(Chymotrypsin, 北京格源天润生物 技术有限公司，医药级)、刀豆蛋白 A(ConA, Sigma, 生化纯)、人免疫球蛋白 G(Human IgG, Sigma, $\geqslant$ 95\%)储备液分别由精确称取 $3.0 \mathrm{mg}$ 各蛋白质溶于 3 
$\mathrm{mL}$ 磷酸盐缓冲液 $(\mathrm{PBS}, 0.01 \mathrm{~mol} / \mathrm{L}, \mathrm{pH}$ 7.4)中配成并 储存于 $4^{\circ} \mathrm{C}$ 冰箱中备用. Britton-Robinson(BR)广泛缓 冲溶液用于调节银胶溶液的 $\mathrm{pH}$ 值; $\gamma$-丙氨基三乙氧 基硅烷(APTES，Sigma，98\%)用于载玻片的硅烷化; $25 \%$ 戊二醛溶液用于在玻片上偶联蛋白质. 柠檬酸三 钠(Sodium Citrate, 成都东金化学试剂有限公司, 分 析纯 $)$ 和硝酸银 $\left(\mathrm{AgNO}_{3}\right.$, 河南桐柏金弘弘银制品有限责 任公司，？99.8\%)用于银纳米粒子的合成. 实验用 水为超纯水.

\section{2 主要仪器}

U-3010 型紫外-可见分光光度计(日立公司, 日 本)、F-4500 型荧光分光光度计(日立公司, 日本)、 SZCL-B 数显智能控温磁力搅拌器(予华仪器公司, 中 国巩义)

\section{$1.3 \mathrm{AgNPs}$ 的制备及表征}

本文采用柠檬酸三钠还原硝酸银法制备银纳米 粒子 ${ }^{[15]}$. 取新配置的 $1.0 \times 10^{-3} \mathrm{~mol} / \mathrm{L}^{\mathrm{AgNO}_{3}}$ 溶液 50 $\mathrm{mL}$ 于洁净的圆底烧瓶中, 将溶液加热至沸腾并使之 保持微沸状态. 在剧烈摚拌下，向溶液中加入 $2 \mathrm{~mL}$ $1 \%$ 柠檬酸三钠溶液. 溶液由无色逐渐变成棕黄色, 10 15 min后停止加热, 继续摚拌至溶液冷却. 将溶 液置于 $4{ }^{\circ} \mathrm{C}$ 冰箱中避光保存, 备用. 取少量 $\mathrm{AgNPs}$ 溶 液于铝铂片上, 自然风干后, 使用扫描电子显微镜 (SEM)对其形貌进行成像; 并在U-3010 型紫外-可见 分光光度计和 F-4500 型荧光分光光度计上测定 $\mathrm{AgNPs}$ 溶液吸收及散射光谱.

\section{4 实验方法}

根据文献[16,17]采用图 1 所示步骤, 分别用饱和 氢氧化钠和浓酸浸泡载玻片使之表面活化而带上羟 基(一OH), 接着在 $2 \%$ APTES的丙酮溶液中浸泡 2 $\min$ 进行硅烷化使其表面带上氨基 $\left(-\mathrm{NH}_{2}\right)$. 用水冲 洗载玻片表面并用氮气吹干后, 在 $120^{\circ} \mathrm{C}$ 烘箱中固定 $30 \mathrm{~min}$. 将载玻片插入 $2.5 \%$ 的戊二醛水溶液偶联 60 $\min$ 使载玻片表面带上的醛基(一 $-\mathrm{CHO})$. 最后将载玻 片插入 $1 \mathrm{~mL}$ 蛋白质溶液中进行蛋白质的固载, 时间 为 $12 \mathrm{~h}$. 将固载有蛋白质的载玻片插入 $\mathrm{AgNPs}$ 溶液中 吸附 $10 \mathrm{~min}$, 用自制装置将载玻片固定在F-4500 型 荧光分光度计上检测载玻片表面吸附的AgNPs的散 射强度并摄像.

\section{2 结果与讨论}

\section{1 蛋白质对 AgNPs 的吸附}

光与金属纳米粒子表面的自由电子相互作用会 引起自由电子发生集体相干振荡, 此便为表面等离 子体共振(SPR). 金、银纳米粒子具有等离子体共振 吸收及散射特性 ${ }^{[18]}$. 由图 2 可知本研究制得的AgNPs 最大等离子体共振吸收在 $410 \mathrm{~nm}$, 最大等离子体共 振散射在 $452 \mathrm{~nm}$, 平均粒径约为 $46 \mathrm{~nm}$. 吸收及散射 均在可见区，其散射光为蓝色，肉眼可见. 随颗粒大 小、形状的变化其吸收及散射也发生改变, 两者与光 的共同作用使银纳米粒子溶液呈现不同的颜色. 图 3 是经处理的玻片在不同浓度的 $\operatorname{IgG}$ 溶液中进行固载,

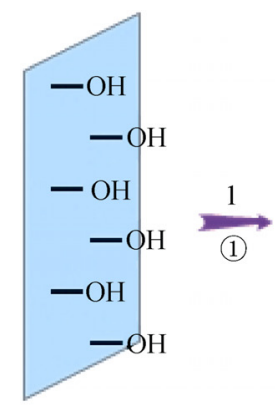

1. APTES

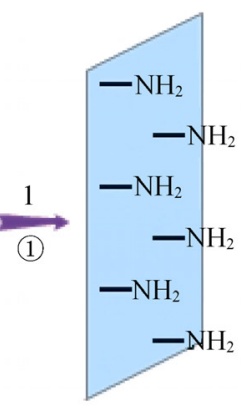

2. 戊二醛

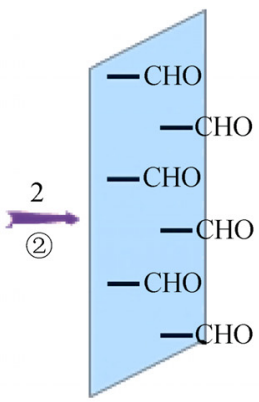

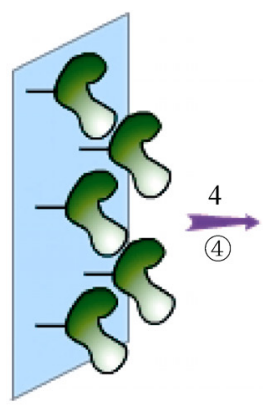

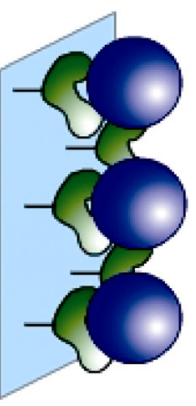

4. 银钠米粒子

图 1 载玻片表面蛋白质的固载及蛋白质对 $\mathrm{AgNPs}$ 的吸附

(1)硅烷化; (2)戊二醛偶联; (3)蛋白质固载; (4) $\mathrm{AgNPs}$ 的吸附 


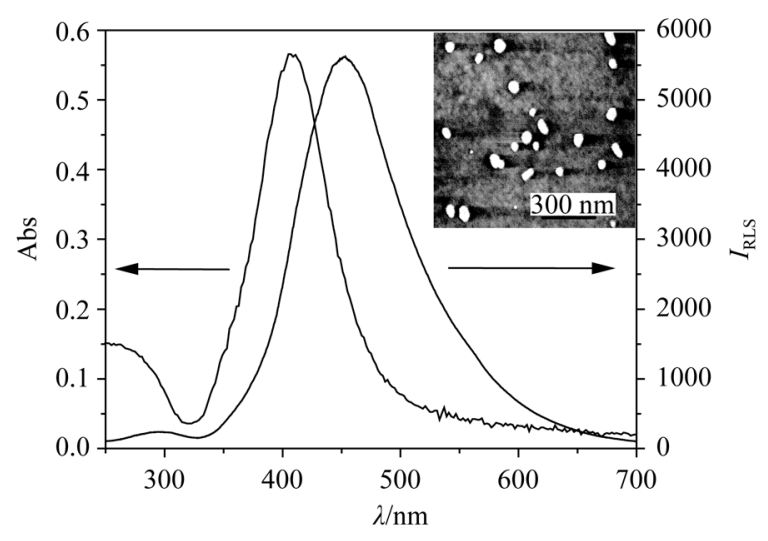

图 $2 A g N P s$ 溶液的吸收及散射光谱图

检测在室温下完成, 光电倍增管电压为 $400 \mathrm{~V}$, 狭缝宽度为 $5 \mathrm{~nm}$. 内置图像为银纳米粒子的扫描电子显微镜(SEM)图

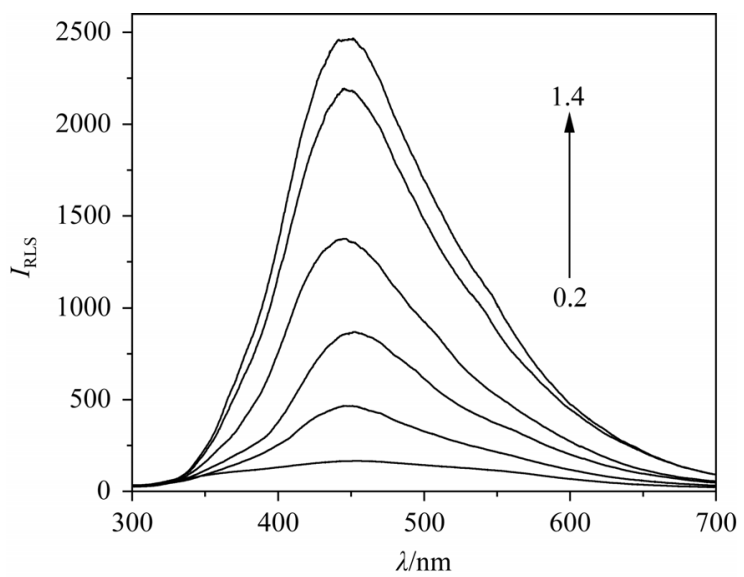

图 3 载玻片在不同浓度 IgG 溶液中固载吸附 AgNPs 后的 散射光谱图

$\mathrm{IgG}$ 的浓度由下到上分别为 $0.2,0.5,0.8,1.0,1.2,1.4 \mu \mathrm{g} / \mathrm{mL}$

吸附 $\mathrm{AgNPs}$ 后检测到的散射光谱图. 从图中可以看 出最大等离子体共振散射强度随 $\mathrm{IgG}$ 的浓度增大而 增大. 这说明在浓度大的溶液中玻片上固载的蛋白 质量大，致使吸附的 $\mathrm{AgNPs}$ 增多.

\section{2 蛋白质对 AgNPs 吸附机理}

实验所制备的 AgNPs 表面包被有柠檬酸根. 因 而在不同 $\mathrm{pH}$ 值溶液中, 蛋白质和柠檬酸根会带有不 同的电荷，两者之间的静电吸附作用随 $\mathrm{pH}$ 值的改变 而发生改变. 故不同 $\mathrm{pH}$ 条件下的蛋白质对 AgNPs 的 吸附不同(图 4). 从图中可以看出, 散射强度随 $\mathrm{pH}$ 值
增大呈先增后减的变化, 说明蛋白质对 AgNPs 的吸 附存在一个最理想的 $\mathrm{pH}$ 值, 使得在该 $\mathrm{pH}$ 值下蛋白 质对 AgNPs 的吸附最强. 当然这种变化也不排除酸 碱引起蛋白质的构象变化.

在酸或碱溶液中，蛋白质会在一定程度上发生 变性，高级结构被破坏，使蛋白质对 AgNPs 的吸附作 用减弱. 当蛋白质处于 $\mathrm{pH} \geqslant \mathrm{p} I_{0}$ 的溶液中时, 由于 同电相斥, 蛋白质不吸附 AgNPs. 但从检测到的散射 信号来看, 仍有 $\mathrm{AgNPs}$ 吸附在蛋白质上面. 我们认为 此时是蛋白质所含半胱氨酸的颈基与银原子形成共 价键, 致使蛋白质对 AgNPs 有一定程度的吸附作用. 不同的蛋白质半胱氨酸的含量不同, 对 AgNPs 的吸 附程度也不同.

图 4 显示出六种蛋白质对 AgNPs 最大吸附强度 是不同的. 人 $\operatorname{IgG}$ 对 AgNPs 的最大吸附明显高出其 他五种蛋白质. 其中主要原因跟蛋白质分子量有关. 蛋白质由 20 种基本氨基酸组成, 其分子量大小与氨 基酸数目一致. 大分子量的 $\mathrm{IgG}$ 含有较前五种蛋白质 更多的氨基酸，相比之下有更多的颈基，同时在最理 想的吸附 $\mathrm{pH}$ 条件下蛋白质带的正电荷最多, 使其对 AgNPs 的吸附作用最强.

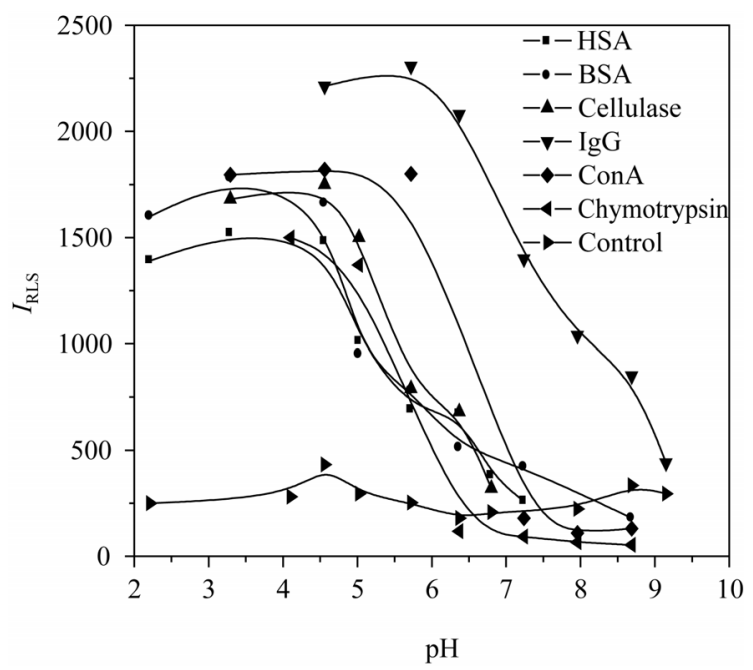

图 4 固载在载玻片上的 6 种蛋白质及空白对照在不同 $\mathrm{pH}$ 条件下对 AgNPs 的吸附

\section{3 蛋白质固载的可视化检测}

经相同步骤处理的载玻片表面带有同样数量的 
醛基. 进行蛋白质固载时, 如果蛋白质质量小于一定 值, 溶液中的蛋白质能全部与醛基作用而被固载在 载玻片表面, 此时玻片表面固载不完全. 如果溶液中 蛋白质质量大于一定值, 溶液中的蛋白质只有部分 与载玻片表面的醛基作用, 即固载完全. 在此我们把 蛋白质这个临界量称为最小完全固载量(LCIQ).

当溶液中的蛋白质质量小于 LCIQ 时, 随溶液中 蛋白质质量的增加, 载玻片上固载的蛋白质也随之 增加. 当溶液中的蛋白质质量大于等于 LCIQ 时, 玻 片完全固载. 在相同的银胶溶液中, AgNPs 吸附量在 一定范围内与固载的蛋白质量成正比. 因此, 通过检 测载玻片上吸附的银纳米粒子的散射强度, 可以间 接说明玻片上蛋白质的固载情况. 图 5 表示的是五种 蛋白质在不同的固载浓度(质量)下固载蛋白吸附银纳 米粒子后玻片的散射强度. 从图中可以看出，随着蛋 白质浓度的增加，玻片的散射强度也随之增大，最后 达到最大值.

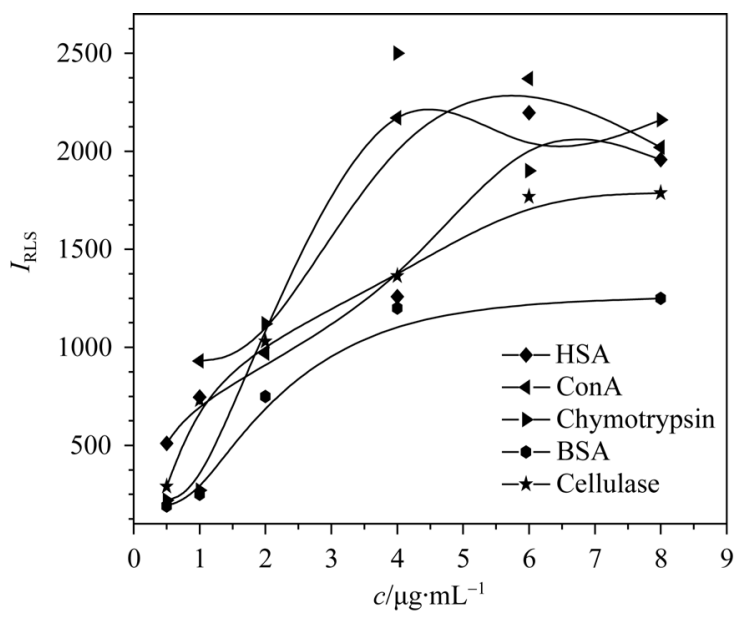

图 5 蛋白质浓度对吸附 AgNPs 的影响

蛋白质浓度分别为 $0.5,1.0,2.0,4.0,6.0,8.0 \mu \mathrm{g} / \mathrm{mL}$

实验表明, 玻片表面的散射强度与玻片上固载 的蛋白质量在一定范围内存在简单函数关系. 我们 考察了在玻片上固载不同量的 $\mathrm{IgG}$ 时, 玻片吸附银纳 米粒子后的散射强度. 如图 6 所示, 人 $\mathrm{IgG}$ 在玻片上 的固载量(即浓度, 蛋白质固载量数值与浓度数值一 致)与其吸附的银纳米粒子的散射强度的对数之间存 在较好的线性关系: $\lg I_{\mathrm{RLS}}=2.05+1.09 c, R=0.995$, 线性范围为 $0.2 \sim 1.2 \mu \mathrm{g} / \mathrm{mL}$. 因此可以通过测定银纳
米粒子的散射强度建立一种检测蛋白质在玻片上固 载量的分析方法. 与Marshall ${ }^{[13]}$ 利用银染法对聚丙烯 酰胺凝胶中BSA的检测获得了很高的灵敏度 $(<0.01$ $\mathrm{ng} / \mathrm{mm}^{2}$ )相比, 本法固载的 BSA 检测可以达到约 1 $\mathrm{ng} / \mathrm{mm}^{2}$. 灵敏度相对较低, 原因是玻片本身的背景及 玻片对银纳米粒子微弱的非特异性吸附作用，但相 对较低的灵敏度却不影响蛋白质最小完全固载量的 可视化检测.

吸附在载玻片上的银纳米粒子有强烈的等离子 共振散射特性，其散射光除了可以使用荧光分光光 度计检测以外，还可以在白光发光二极管(LED)电筒 照射下实现肉眼可见. 图 6 内的照片显示固载不同量 $\mathrm{IgG}$ 的玻片吸附银胶后，在 LED 电筒照射下吸附在玻 片上的银纳米粒子发出强烈的蓝色散射光. 当 $\mathrm{IgG}$ 固 载量较小时，银胶在玻片上吸附量少，而且不均匀; 当 $\mathrm{IgG}$ 的固载量增大时, 银纳米粒子的吸附增多, 且 逐渐变的均匀, 最后几乎不再变化. 基于此可以建立 可视化检测 LCIQ 的方法. 从图 6 照片中 AgNPs 的 散射光可以看出, 当 $\mathrm{IgG}$ 的量小于 $1.2 \mu \mathrm{g}$ 时, 载玻片 上没有完全均匀吸附上 $\mathrm{AgNPs}$, 即玻片固载不完全. 而当 $\mathrm{IgG}$ 的量大于等于 $1.2 \mu \mathrm{g}$ 时, 玻片上均匀吸附有 AgNPs, 蓝色的散射光均匀清晰可见, 此时固载完全. 故我们可以确定 $\mathrm{IgG}$ 在约 $1 \mathrm{~cm}^{2}$ 载玻片两面上的最小 完全固载量为 $1.0 \mu \mathrm{g} \leqslant \mathrm{LCIQ}<1.2 \mu \mathrm{g}$.

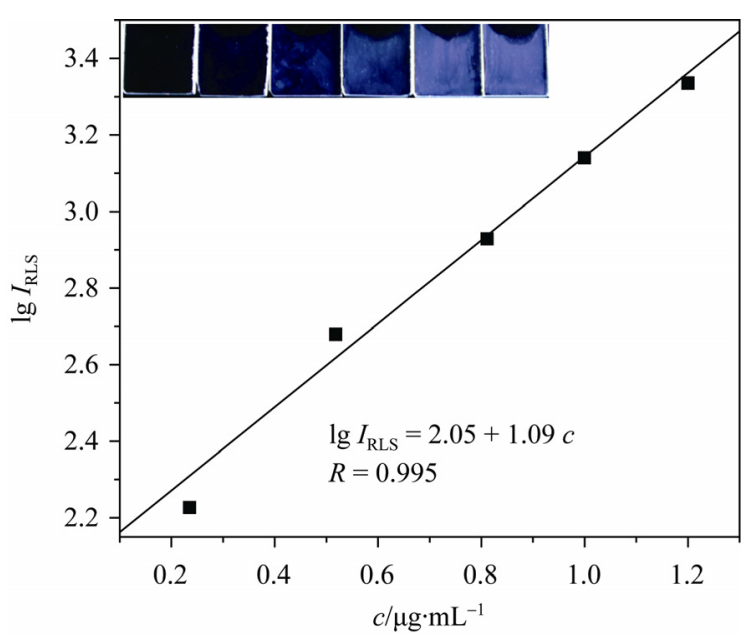

图 6 散射强度对数与浓度的线性关系

内嵌图的前五张玻片为对应载玻片在白光照射下的摄像图, 第六 张玻片对应的固载浓度为 $1.4 \mu \mathrm{g} / \mathrm{mL}$ 


\section{3 结论}

上述结果表明, 蛋白质吸附 AgNPs 的机理涉及 到静电吸附和共价吸附. 当蛋白质处于 $\mathrm{pH}<\mathrm{p} I_{0}$ 的溶 液中时，带正电荷的蛋白质与带负电荷的 AgNPs 间
发生静电吸附作用. 颈基与银原子之间能形成共价 键，所以蛋白质与 AgNPs 之间还存在颈基的吸附作 用. 我们根据固载不同量蛋白质吸附 AgNPs 后散射 光的可见情况建立可视化检测蛋白质在载玻片上固 载的方法.

致谢本工作得到国家自然科学基金(批准号: 20425517)资助.

\section{参考文献}

1 间海刚. 血清白蛋白与银纳米粒子的相互作用及包覆. 漳州师范学院学报(自然科学版), 2003, 16(4): 54一 58

2 Jing C, Fang Y. Experimental (SERS) and theoretical (DFT) studies on the adsorption behaviors of L-cysteine on gold/silver nanoparticles. Chem Phys, 2007, 332: 27-32[DOI]

3 Yguerabide J, Yguerabide E E. Light-scattering submicroscopic particles as highly fluorescent analogs and their use as tracer labels in clinical and biological applications. I . Theory. Anal Biochem, 1998, 262: 137-156[DOI]

4 Huang C Z, Li Y F, Mao J G, Tan D G. Determination of protein concentration by enhancement of the preresonance light-scattering of $\alpha, \beta, \gamma, \delta$-tetrakis(5-sulfothienyl)porphine. Analyst, 1998, 123: 1401-1406[DOI]

5 Huang C Z, Li K A, Tong S Y. Determination of nucleic acids by a resonance light-scattering technique with $\alpha, \beta, \gamma, \delta$-tetrakis[4-(trimethyl ammoniumyl)phenyl]porphine. Anal Chem, 1996, 68: 2259-2263[DOI]

6 Long Y F, Huang C Z, Li Y F. Hybridization detection of DNA by measuring organic small molecule amplified resonance light scattering signals. J Phys Chem B, 2007, 111: 4535-4538[DOI]

7 Luo H Q, Liu S P, Li N B, Liu Z F. Resonance rayleigh scattering, frequency doubling scattering and second-order scattering spectra of the heparin-crystal violet system and their analytical application. Anal Chim Acta, 2002, 468: 275-286[DOI]

8 Wu L P, Li Y F, Huang C Z, Zhang Q. Visual detection of sudan dyes based on the plasmon resonance light scattering signals of silver nanoparticles. Anal Chem, 2006, 78: 5570-5577[DOI]

9 Yguerabide J, Yguerabide E E. Light-scattering submicroscopic particles as highly fluorescent analogs and their use as tracer labels in clinical and biological applications. II . Experimental characterization. Anal Biochem, 1998, 262: 157-176[DO]

10 El-Sayed I H, Huang X H, El-Sayed M A. Surface plasmon resonance scattering and absorption of anti-EGFR antibody conjugated gold nanoparticles in cancer diagnostics: Applications in oral cancer. Nano Lett, 2005, 5: 829-834[DOI]

11 Huang X H, El-Sayed I H, Qian W, El-Sayed M A. Cancer cell imaging and photothermal therapy in the near-infrared region by using gold nanorods. J Am Chem Soc, 2006, 128: 2115-2120[DOI]

12 蒋治良, 陈媛媛, 梁爱惠, 陶慧林, 唐宁莉, 钟福新. 痕量纤维蛋白原的银纳米标记免疫共振散射光谱分析. 中国科学 B 辑: 化学, 2006, 36(5): 419-424

13 Marshall T. Detection of protein in polyacrylamide gels using an improved silver stain. Anal Biochem, 1984, 136: 340-346[DOI]

14 斯佩克特 D L，戈德曼 R D, 莱因万德 L A , 著. 黄培堂, 等译. 细胞实验指南(上册). 北京: 科学出版社, 2001. 509一510

15 Lee P C, Meisel D. Adsorption and surface-enhanced raman of dyes on silver and gold sols. J Phys Chem, 1982, 86: 3391 - 3395[DOI]

16 Zhao H W, Huang C Z, Li Y F. A novel optical immunosensing system based on measuring surface enhanced light scattering signals of solid supports. Anal Chim Acta, 2006, 564: 166-172[1DOI]

17 Ling J, Huang C Z, Li Y F. A label-free visual immunoassay on solid support with silver nanoparticles as plasmon resonance scattering indicator. Anal Biochem, 2008, 383: 168-173[DOI]

18 Jain P K, Huang X H, El-Sayed I H, El-Sayed M A. Review of some interesting surface plasmon resonance-enhanced properties of noble metal nanoparticles and their applications to biosystems. Plasmonics, 2007, 2: 107-118[DOI] 